\title{
Functional gastrointestinal disorders in newborns: nutritional perspectives
}

\author{
Roberto Bellù, Manuela Condò \\ Neonatal Intensive Care Unit, Manzoni Hospital, Lecco, Italy
}

\begin{abstract}
Functional gastrointestinal disorders (FGIDs) definition in children has changed over the years trying to facilitate clinicians, because the diagnostic process is complicated by the interpretation variability of symptoms described by children or by their caregivers for newborns and toddlers. This review refers to the Rome IV classification system, drafted in 2016. FGIDs pathophysiology is multifactorial and still poor understood, with limitations for the therapeutic process, which results often in unnecessary and alternative treatments trying to reduce the relevant caregiver distress, but with increased costs for families and for the National Health Service. This study reports the most recent evidence-based treatments for FGIDs in newborns: though in most cases the first action is an educational and behavioral intervention, reassuring caregivers about the transient and self-limiting natural history of FGIDs, there is now more evidence to recommend probiotics in some infant FGIDs.
\end{abstract}

\section{Introduction}

Functional gastrointestinal disorders (FGIDs) refer to a wide group of signs and symptoms, that are chronic or recurrent and cannot be explained by structural or biochemical gastrointestinal abnormalities. ${ }^{1}$

Correspondence: Roberto Bellù, Neonatal Intensive Care Unit, Manzoni Hospital, Via Eremo 9, 23900 Lecco, Italy.

Tel.: +39.0341.489231 - Fax: +39.0341.489247.

E-mail: r.bellu@asst-lecco.it

Key words: Functional gastrointestinal disorders; FGIDs; Newborn; Infant.

Contributions: the authors contributed equally.

Conflict of interest: the authors declare no potential conflict of interests.

Funding: funded by Merqurio Editore S.r.L, with the unconditional contribution of Nestlè Italiana S.p.A.

Received for publication: 19 April 2018.

Accepted for publication: 20 April 2018.

This work is licensed under a Creative Commons Attribution

NonCommercial 4.0 License (CC BY-NC 4.0).

(C) Copyright R. Bellù and M. Condò, 2018

Licensee PAGEPress, Italy

La Pediatria Medica e Chirurgica 2018; 40:198

doi:10.4081/pmc.2018.198
One of the great problems is the diagnostic process that featured FGIDs over the past decades, most due to the difficulty for clinicians to classify symptoms described by children, for instance discerning them from emotional reactions; for newborns and toddlers, the variability of diagnostic interpretation increases more and more because the anamnestic information is reported by caregivers. The process of standardization of functional gastrointestinal disorders was possible through the constitution of working teams of experts during international gastroenterology meetings, beginning from adults and then specifying diagnostic criteria for children. This classification system was updated over the years till the formulation of the latest Rome IV criteria in 2016. ${ }^{2}$ The Rome Foundation proposed guidelines through literature review and a consensus process not only for FGIDs diagnosis, but also for treatment, referring more on children symptoms and less on physiological alterations, like motility. ${ }^{3}$ Compared to previous criteria, clinician's approach is more individualized, also due to innovative materials: a software program (interactive clinical decision toolkit) matches diagnostic algorithms with a case-based record (multi-dimensional clinical profile). In addition there is attention to the neuro-intestinal interactions, in particular versus the factors involved in the origin and perception of pain. ${ }^{2}$

Among pediatric patients FGIDs have a different distribution by age and in particular from birth till the first year of life they include: infant regurgitation, infant rumination syndrome, cyclic vomiting syndrome, infant colic, functional diarrhea, infant dyschezia and functional constipation. ${ }^{1}$

Despite the improvements on FGIDs knowledge, the pathophysiologic mechanisms of these functional disorders in children are still poor understood. Experts agree to a multifactorial hypothesis, concerning genetic and psychological predisposition, dysregulation of pain routes, stress, ambient factors and gut microbiota. The following result is that there are limitations to find the most proper therapeutic approach. ${ }^{2}$

A recent systematic review emphasizes the relevant costs of FGIDs in England and its underestimation also due to the use of alternative treatments. ${ }^{4}$

The aim of this study is to review the most recent evidencebased treatments for functional gastrointestinal disorders in newborns, classified according to Rome IV criteria, with particular attention to nutritional aspects.

\section{Functional gastrointestinal disorders in newborns}

Limiting the overview of FGIDs during the neonatal period and according to the recent Rome IV diagnostic criteria, in this study we consider the following disorders: infant regurgitation, infant colic, infant dyschezia and functional constipation. Their worldwide prevalence varies from about $15 \%$ to $30 \%$, recognizing infant regurgitation and functional constipation as the most prevalent in infant and toddlers. ${ }^{4-7}$ 
The first impact of FGIDs in newborns is on the diagnostic process, which is based on physical examination and on medical history through parental reports. In particular, it is to consider the negative effect of these disorders on the family quality of life and how it can affect parental perception of the newborn's symptoms. Furthermore, infant symptoms of FGIDs can occur in combination. 1,2,5

Though functional gastrointestinal disorders are self-limiting and symptoms are transient, the distress for family and clinicians are such to cause many unmotivated dietetic changes, sometimes stopping breastfeeding or turning to another formula in formulafed infants. $1,2,5$

In order to underline the current knowledge of FGIDs and its limits in the first month of life, first we report the definition of each of the aforementioned conditions, with the presentation possibilities and the pathophysiology as reported in Rome IV criteria. The following section is dedicated to treatment options for each functional disorder.

\section{Infant regurgitation}

Infant regurgitation is very common and happens in healthy infants between 3 weeks and 12 month of age. It refers to the possibility to see the involuntary reflux of the gastric content. Diagnostic criteria include the presence of regurgitations at least two times per day, for at least three weeks. Moreover it is necessary the absence of retching, hematemesis, aspiration, apnea, failure to thrive, feeding or swallowing difficulties and abnormal posturing. It has to be noted that there is no more mention of troublesome symptoms: in the precedent criteria it was added to differentiate gastroesophageal reflux disease (GERD) from uncomplicated regurgitation, but it is difficult to quantify.

Among the underlying pathophysiologic mechanisms there are anatomical (small esophageal dimensions), postural and behavioral factors (overfeeding). $1,2,5$

\section{Infant colic}

Infant colic refers to infants before five months of life, including in this period the beginning and the end of symptoms. A systematic review and meta-analysis in 2017 reported a drop in fussing and crying duration after 8-9 weeks of age, but colic was defined using modified Wessel's criteria (Wessel rule of three: crying for $>3$ hours per day, for $>3$ days per week, for 3 weeks). 8,9

In the definition for clinical purpose, caregiver report is about recurrent and prolonged periods of crying, fussing or irritability, which cannot be prevented or resolved by caregivers and that cannot be explained by other causes (no evidence of fever, illness, or failure to thrive). Fussing is specified as an intermittent distressed vocalization, a behavior that is not quite crying and that is difficult to discern from crying, because infants often alternate the two symptoms. The prevalence of colic in infants is about $20 \%$, but it depends on parental perception of crying. Also for this reason there is no mention of the modified Wessel's criteria, because duration of infant crying is considered arbitrary and influenced by cultural factors and data collection is impractical. The pathophysiology of this disorder is still unknown, but it is considered part of the normal developmental process, with a normal variability that includes different expression of crying. Again, the aforementioned caregivers' perceptions of crying play a crucial role.1,2,5 Other hypothesis concern neurological and intestinal immaturity, intestinal hypermotility and dysbiosis and allergy to cow's milk proteins. ${ }^{10-13}$

\section{Infant dyschezia}

Infant dyschezia is a condition that concerns infants less than 9 months of age and it is characterized by infant straining and crying for at least 10 minutes before the release or not of soft stools, without the presence of other illnesses. Dyschezia is often misunderstood with constipation, but the pathophysiologic mechanism is represented by incoordination between increased intra-abdominal pressure and relaxation of the muscular pelvic floor during defecation.

\section{Functional constipation}

Functional constipation arises from the association between painful defecation and stool retention, with a possible self-sustaining system, because the infant avoids defecation to not feel pain. Prevalence varies from 5\% and 27\%. It develops in infants up to 4 years of age and its definition according to Rome IV criteria includes the presence of at least two the following features for one month: two defecations or less per week, history of marked stool retention, or of painful bowel movements, or of big volume stools, and the presence of a large rectal fecal mass. ${ }^{1,2,4,5,14,15}$ There is still heterogeneity and inconsistency to define functional constipation in children, as reported by a recent systematic review. ${ }^{16}$

\section{Treatment interventions of functional gastrointestinal disorders in newborns}

\section{Infant regurgitation}

Referring to infant regurgitation the first thing to know for clinicians and to underline to the infant caregivers is that it is a transient disorder, resolving within the first year of life in the $90 \%$ of cases. No medical treatment is recommended, in particular there is no evidence of benefit from the use of proton pump inhibitors, neither in GERD. ${ }^{17-19}$ Intervention consists overall in reassuring caregivers, explaining the natural history of the condition. There is no reason to stop breastfeeding, but nutritional choices include smaller and thickened feeding and anti-regurgitation formulas. ${ }^{20-22}$ There is some evidence that prebiotics and probiotics could prevent infant regurgitation by increasing gastric emptying.

Urbanska et al. reviewed in 2014 the efficacy of Lactobacillus reuteri DSM 17938 in infants and children finding possible evidence, even if limited, in reducing regurgitation in infant with gastroesophageal reflux and efficacy in preventing regurgitation in breast-fed and formula-fed infants. It was anyway recommended result replication and the need of safety data for its use. ${ }^{23}$

Among conservative options there is postprandial positioning in lateral, prone and anti-Trendelenburg posture, but because of the increased risk of sudden infant death syndrome (SIDS), it is recommend from the American Academy of Pediatrics to sleep in the supine position. $1,5,24$

\section{Infant colic}

One intervention in infant colic is reassuring and giving support to the infant caregivers, because this kind of disorder can be frustrating and anxiogenic for the family and can undermine parental confidence about infant care, in particular for nutritional aspects. Conversely, there is no recommendation for pharmacologic treatments. Rome IV guidelines report controversial evidence for nutritional interventions: probiotics, hydrolysed formula for formula-fed infants, cows' milk free diet for breastfeeding mothers and herbal medicine (fennel, peppermint).

Regarding probiotics, many systematic reviews investigated their efficacy in the management or the prevention of infant colic, mostly concluding that, even if a specific strain of probiotic ( $L$. reuteri) was effective in reducing infant crying in colic breastfed infants, the limits of the study in terms of heterogeneity and 
methodological issues were such to not support the general use of probiotics. ${ }^{25,26}$ Several studies were conducted in particular on the effects of L. reuteri strain DSM 17938 in infant colic, assuming that this functional disorder could be secondary to a modified gut microbiota, causing intestinal inflammation.27-29 Many authors reported clinical efficacy of $L$. reuteri in colic breastfed infant. $30-35$ and more recently Savino et al. found in a randomized controlled trial a beneficial effect of this probiotic in decreasing crying time and in reducing fecal calprotectin, a marker of intestinal inflammation, in healthy term infants fed with exclusive or predominant breast milk and suffering from colic. ${ }^{36}$ Fatheree et al. 37 excluded severe adverse effects of $L$. reuteri strain DSM 17938 in colic breastfed newborns, but observed no significant change in crying time or inflammatory biomarkers, as previously reported. ${ }^{38}$

A systematic review of infant colic interventions in breastfed infants younger than 6 months was conducted in 2016, using Wessel or modified Wessel criteria. Probiotics containing L. reuteri was effective, unlike simethicone; also preparations containing fennel resulted effective, but had to be considered with caution because of methodological problems and the high risk of bias. ${ }^{39}$ Finally we report the results of a systematic review with network meta-analysis published in 2017, which confirm the superiority of L. reuteri DSM 17938 in reducing the duration of crying time in infant colic with less than 6 month of age. Secondly, specialized infant formula (partially hydrolyzed, whey-protein derivatives) were the most effective interventions, while for other interventions (drugs, herbals, acupuncture, massage) there was low evidence to be recommended. For the less effective treatment (reassurance/education), the authors speculated the effect of high risk of bias in the related trials. This study is innovative because overcomes previous study limitations, related to great heterogeneity and risk of bias of clinical trials on this topic, using the network-meta-analysis approach. 40

\section{Infant dyschezia}

Even for infant dyschezia it is not necessary any medical treatment, in particular laxatives. It is not recommended to make anal stimulation, which could induce behavioral disturbances in infants, like waiting stimulation to release stools. ${ }^{1,5}$ An important intervention consists in reassuring caregivers that it is a disorder resolving spontaneously and that can benefit from educational procedures, teaching infants to coordinate abdominal push down with pelvic relaxation.

\section{Functional constipation}

In functional constipation interventions consist in education of infants and caregivers, reducing fear and assuring a painless stool release until the disorder resolves. On the other hand, conclusive data about the effect of dietetic products and laxatives are still lacking. 1,5 ESPGHAN (European Society for Pediatric Gastroenterology, Hepatology and Nutrition) and NASPGHAN (North American Society for Pediatric Gastroenterology, Hepatology and Nutrition) recommendations provided in 2014 do not support the use of fiber, extra fluid intake, prebiotics and probiotics; among oral laxatives polyethylene glycol (PEG) and enemas are effective for fecal disimpaction. 41

\section{Conclusions}

This review remarks on one side the lack of proper treatments in some of the FGIDs expressing during the neonatal period, but also the recent confirming evidence of effective nutritional intervention as for probiotics in infant colic, overcoming some of the previous controversial results. However, the difficulty of standardizing FGIDs definition persists, because classification criteria have changed over the years to improve the diagnosis and treatment process for clinicians; different FGIDs definitions still coexist making more difficult the comparison among clinical trials with the usual statistical techniques.

Furthermore, it is important to emphasize the vulnerable age of infants, in particular during the neonatal period, because they are not able to fully express their symptoms and because they totally depend by their caregivers, increasing more the distress and restlessness deriving from infant functional gastrointestinal disorders. As parental frustration undermines infant care ability, just for essential needs like nutritional aspects, it could represent a very dangerous vicious cycle putting at risk the psycho-physical wellbeing of the whole family. Just think to the possible impact of renouncing to breastfeeding during the neonatal life or more dangerously to the traumatic effects of shaken-baby syndrome.

For this reason, waiting for more knowledge of the pathophysiologic mechanisms of FGIDs in infants and consequently for improvement of treatment options, it is important first of all facilitating the diffusion of the new nutritional evidences into the clinical practice for the applicable disorders (L. reuteri DSM 17938 in infant colic). For the other FGIDs, lacking of such evidence-based options, we think it is mandatory to optimize caregiver support. In this regard, National Health Service could prevent and reduce the social impact of such troublesome disorders, providing more counsellors and psychologists next to clinicians and families in hospital and outpatient structures. They could be also useful counselling courses for clinicians, improving their listening capacity, with a better comprehension of the infant family needs.

We speculate a secondary positive economic impact for reduction in medical prescriptions or in self-parental decision of unnecessary treatments.

Updated guidelines and innovative materials help clinicians to have a more individualized approach for the management of functional gastrointestinal disorders in children. Unlike others ages, in newborns medical history is reported by caregivers, so medical variability is affected also by parental perceptions of infant symptoms. Despite progress on FGIDs diagnostic process, their pathophysiologic mechanisms are still little known, causing limitations to the therapeutic approach. Since one marker of FGIDs in infants is caregiver distress and anxiety, reducing parental confidence about infant care, we underline the importance of educational and behavioral interventions to support infant caregivers, limiting also the costs of ineffective treatments. On the other hand, while effective pharmacologic treatments are mostly lacking, among nutritional interventions there is more recent evidence about the efficacy of probiotics in some infant FGIDs.

\section{References}

1. Benninga MA, Nurko S, Faure C, et al. Childhood functional gastrointestinal disorders: neonate/toddler. Gastroenterology 2016;150:1443-55.

2. Koppen IJN, Nurko S, Saps M, et al. The pediatric Rome IV criteria: what's new? Exp Rev Gastroenterol Hepatol 2017;11:193-201.

3. Drossman DA. Functional gastrointestinal disorders: history, pathophysiology, clinical features, and Rome IV. Gastroenterology 2016;150:1262-79.

4. Mahon J, Lifschitz C, Ludwig T, et al. The costs of functional gastrointestinal disorders and related signs and symptoms in 
infants: a systematic literature review and cost calculation for England. BMJ Open 2017;7:e015594

5. Zeevenhooven J, Koppen IJN, Benninga MA. The new Rome IV criteria for functional gastrointestinal disorders in infants and toddlers. Pediatr Gastroenterol Hepatol NUTR 2017;20:1-13.

6. Ferreira-Maia AP, Matijasevich A, Wang YP. Epidemiology of functional gastrointestinal disorders in infants and toddlers: a systematic review. World J Gastroenterol 2016;22:6547-58.

7. Robin SG, Keller C, Zwiener R, et al. Prevalence of pediatric functional gastrointestinal disorders utilizing the Rome IV criteria. J Pediatr 2018;195:134-9.

8. Wolke D, Bilgin A, Samara M. Systematic review and metaanalysis: fussing and crying durations and prevalence of colic in infants. J Pediatr 2017; 185:55-61.e4.

9. Wessel MA, Cobb JC, Jackson EB, et al. Paroxysmal fussing in infancy, sometimes called colic. Pediatrics 1954;14:421-35.

10. Savino F. Focus on infantile colic. Acta Paediatr 2007;96: 1259-64.

11. Camilleri M, Park SY, Scarpato E, Staiano A. Exploring hypotheses and rationale for causes of infantile colic. Neurogastroenterol Motil 2017;29.

12. Gensollen T, Iyer SS, Kasper DL, Blumberg RS. How colonization by microbiota in early life shapes the immune system. Science 2016;352:539-44.

13. Lu CY, Ni YH. Gut microbiota and the development of pediatric diseases. J Gastroenterol 2015;50:720-6.

14. Van Tilburg MA, Hyman PE, Walker L, et al. Prevalence of functional gastrointestinal disorders in infants and toddlers. J Pediatr 2015;166:684-9.

15. Chogle A, Velasco-Benitez CA, Koppen IJ, et al. A populationbased study on the epidemiology of functional gastrointestinal disorders in young children. J Pediatr 2016;179:139-43.

16. Kuizenga-Wessel S, Heckert SL, Tros W, et al. Reporting on outcome measures of functional constipation in children- a systematic review. JPGN 2016;62:840-6.

17. Orenstein SR, Hassal E, Furmaga-Jablonska W, et al. Multicenter, double-blind, randomized, placebo-controlled trial assessing the efficacy and safety of proton pump inhibitor lansoprazole in infants with symptoms of gastroesophageal reflux disease. J Pediatr 2009;154:514-20.

18. Rosen R. Gastroesophageal reflux in infants: more than just a phenomenon. JAMA Pediatr 2014;168:83-9.

19. Vandenplas Y, Rudolph CD, Di Lorenzo C, et al. Pediatric gastroesophageal reflux clinical practise guidelines: joint recommendations of the North American Society for Pediatric Gastroenterology, Hepatology and Nutrition (NASPGHAN) and the European Society for Pediatric Gastroenterology, Hepatology and Nutrition (ESPGHAN). J Pediatr Gastroenterol Nutr 2009;49:498-547.

20. Vandenplas Y, Leluyer B, Cazaubiel M, et al. Double-blind comparative trial with 2 antiregurgitation formulae. J Pediatr Gastroenterol Nutr 2013;57:389-93.

21. Van Wijk MP, Benninga MA, Davidson GP, et al. Small volumes of feed can trigger transient lower esophageal sphincter relaxation and gastroesophageal reflux in the right lateral position in infants. J Pediatr 2010;156:744-8.

22. Horvath A, Dziechciarz P, Szajewska H. The effect of thickened-feed interventions on gastroesophageal reflux in infants: systematic review and meta-analysis of randomized, controlled trial. Pediatrics 2008;122:e1268-77.

23. Urbanska M, Szajewska H. The efficacy of Lactobacillus reuteri DSM 17938 in infants and children: a review of the current evidence. Eur J Pediatr 2014;173:1327-37.

24. Moon RY, Task Force on Sudden Infant Death Syndrome.
SIDS and other sleep-related infant death: expansion of recommendations for a safe infant sleeping environment. Pediatrics 2011;128:e1341-67.

25. Sung V, Collett S, de Gooyer T, et al. Probiotics to prevent or treat excessive infant crying: systemtic review and meta-analysis. JAMA Pediatr 2013;167:1150-7.

26. Anabrees J, Indrio F, Paes B, Alfaleh K. Probiotics for infantile colic: a systematic review. BMC Pediatrics 2013;13:186.

27. Rhoads JM, Fatheree NY, Norori J, et al. Altered fecal microflora and increased fecal calprotectin in infants with colic. J Pediatr 2009;155:823-8,e1.

28. Pärtty A, Kalliomäki M, Endo A, et al. Compositional development of Bifidobacterium and Lactobacillus microbiota is linked with crying and fussing in early infancy. PLoS ONE 2012;7:e32495.

29. Xu M, Wang J, Sun N, et al. The efficacy and safety of the probiotic bacterium Lactobacillus reuteri DSM 17938 for infantile colic: a meta-analysis of randomized controlled trials. PLoS ONE 2015;28:10:e141445.

30. Biagioli E, Tarasco V, Lingua C, et al. Pain-relieving agents for infantile colic. Cochrane Database Syst Rev 2016;9: CD009999.

31. Roos S, Dicksved J, Tarasco V, et al. 454 Pyrosequencing analysis on faecal samples from a randomized DBPC trial of colicky infants treated with Lactobacillus reuteri DSM 17938. PLoS ONE 2013;8:e56710.

32. Savino F, Cordisco L, Tarasco V, et al. Lactobacillus reuteri DSM 17938 in infantile colic: a randomized, doubleblind, placebo-controlled trial. Pediatrics 2010;126:e526-33.

33. Chau K, Lau E, Greenberg S, et al. Probiotics for infantile colic: a randomized, double-blind, placebo controlled trial investigating Lactobacillus reuteri DSM 17938. J Pediatr 2015;166:74-8.

34. Szajewska H, Gyrczuk E, Horvath A. Lactobacillus reuteri DSM 17938 for the management of infantile colic in breastfed infants: a randomized, double-blind, placebo-controlled trial. J Pediatr 2013;162:257-62.

35. Francavilla R, Cristofori F, Indrio F. Indications and recomendations by societies and institutions for the use of probiotics and prebiotics in paediatric functional intestinal disorders. JPGN 2016;63:S36-7.

36. Savino F, Garro M, Montanari P, et al. Crying time and RORg/FOXP3 expression in Lactobacillus reuteri DSM17938treated infants with colic: a randomized trial. J Pediatr 2018;192:171-7.

37. Fatheree NY, Liu Y, Taylor CM, et al. Lactobacillus reuteri for infants with colic: a double-blind, placebo-controlled, randomized clinical trial. J Pediatr 2017;191:170-8.e2.

38. Sung V, Hiscock H, Tang ML, et al. Treating infant colic with the probiotic Lactobacillus reuteri: double blind, placebo controlled randomised trial. BMJ 2014;348:g2107.

39. Harb T, Matsuyama M, David M, Hill RJ. Infant colic-what works: a systematic review of interventions for breast-fed infants. J Pediatr Gastroenterol Nutr 2016;62:668-86.

40. Gutiérrez-Castrellòn P, Indrio F, Bolio-Galvis A, et al. Efficacy of Lactobacillus reuteri DSM 17938 for infantile colic: systematic review with network meta-analysis. Medicine (Baltimore) 2017;96:e9375.

41. Tabbers MM, Di Lorenzo C, Berger MY, et al. Evaluation and treatment of functional constipation in infants and children: evidence-based recommendations from ESPGHAN and NASPGHAN. JPGN 2014;58:258-74. 